\title{
On Death Numbers and Survival Times of Single Dye Molecules
}

\author{
Stefan Wennmalm and Rudolf Rigler* \\ Department of Medical Biophysics, Karolinska Institute, S-171 77, Stockholm, Sweden
}

Received: August 20, 1998; In Final Form: January 20, 1999

\begin{abstract}
In fluorescence measurements on the single molecule level the photochemical stability of the fluorophore is an important factor. We measured in 102 cases the photodestruction of individual tetramethylrhodamine molecules linked to a 217-bp DNA sequence which was attached to a streptavidin-coated glass surface. The fluorophores were excited by a green $\mathrm{HeNe}$ laser at $543.5 \mathrm{~nm}$. For each molecule the survival time (time during which fluorescence can be observed), the fluorescence intensity, and the death number (number of detected photons before decomposition) were registered. On the basis of the analysis of single molecule observations, an exponential distribution of the death numbers and survival times of the 102 molecules was found.
\end{abstract}

\section{Introduction}

By the analysis of single molecules it is possible to obtain information about phenomena that cannot be revealed in bulk measurements. ${ }^{1}$ Studies of conformational fluctuations ${ }^{2-4}$ and of diffusional trajectories in bilayers ${ }^{5}$ are examples of single molecule studies where photostable dye molecules with high absorption cross sections such as rhodamine dyes have been used. Also the possibility to sequence DNA at the single molecule level depends on the photostability of the dye-labeled nucleotides. ${ }^{6,7}$ For the analysis of molecular behavior on the single molecule level the survival time of the fluorescent tag limits the observation time and therefore the whole analysis.

We have analyzed the spectroscopic lifetimes of single tetramethylrhodamine (TMR) molecules which have been used as a sensor for conformational fluctuations in nucleic acids. ${ }^{2-4}$ The time is measured during which a single TMR molecule can be observed (survival time) when linked to a 217-bp DNA sequence which itself is bound to a streptavidinized glass surface by a biotin tag at the $5^{\prime}$-end. We observe that the random time after which a molecule dies is exponentially distributed.

\section{Materials and Methods}

A 217-bp DNA product labeled with a tethered TMR molecule on one end and a biotin molecule at the other end was obtained with the PCR. M13 mp18(+) strand DNA (7250 bases) was used as template in the PCR. 5'-(TMR)-AAAGGGGGATCTGCTGCAAGGCG was used as forward primer (TMR was linked to the primer through a 6-carbon-atom linker) and biotin-labeled 5'-(Bi)-GCTTCCGGCTCGTATGTTGTGTG was used as reversed primer. Streptavidin-coated coverslips were prepared, and through the biotin-streptavidin interaction the biotin end of the sample molecules was bound to the coverslips. For PCR conditions, control analysis of the PCR product, preparation of streptavidin-coated coverslips, and binding of DNA to coverslips, see ref 2.

The experimental setup for the confocal detection of single molecules ${ }^{8,9}$ and fluorescence correlation spectroscopy (FCS) as well as fluorescence decay (FD) measurements has been

* Author to whom correspondence should be addressed. described elsewhere. ${ }^{8-10}$ A Zeiss 40x NA 0.9 objective was used providing a $1 \mu \mathrm{m}$ diameter and $4 \mu \mathrm{m}$ length of the volume element. The total fluorescence detection efficiency of the setup was $(2 \pm 1) \%$. A scanning table (ITK Lahnau, Germany; MC2000) was used to move the cover slip relative to the volume element. The table was moveable in three dimensions with 1 $\mu \mathrm{m}$ steps. A computer program was written to control the table so that a large number of measurements were carried out automatically. A random point on the surface was moved into the volume element after which the $10 \mathrm{~s}$ intensity measurement was immediately started. When the measurement was finished, the table moved the next point into the volume element and so on. Due to reflection of excitation light at the glass-water interface the high signal-to-noise ratio of 1000:1 obtainable in solution $^{8-10}$ could not be reached. The excitation volume was positioned with a vertical distance of $2 \pm 1 \mu \mathrm{m}$ from the glass surface in order to reduce detection of reflected light. The detected background intensity varied at different measuring spots with a magnitude up to the fluorescence intensity of a single molecule. Fluorescence intensity measurements were taken with a digital correlator (ALV, Germany; model ALV-5000).

To reduce the decomposition rate of the fluorophore a laser intensity such that the average count rate of a TMR molecule did not exceed $3 \mathrm{kHz}$ was used. Fluorescence intensity was registered during fixed time intervals of $10 \mathrm{~s}$ with an intermediate delay of $3 \mathrm{~s}$. Because of this it was not possible to detect decompositions in the interval 10-13 s.

For parametrization of histograms the Marquardt-Levenberg nonlinear least-squares algorithm was applied. ${ }^{11}$

\section{Results}

Single Molecule Measurements. In typical single molecule measurements the fluorescence intensity is constant until it spontaneously drops to the background level (Figure 1A-C). Each measurement gives the survival time, fluorescence intensity, and total number of photons detected before decomposition for a single molecule. Inspection of many single molecule traces suggests that the time of breakdown and the number of photons detected before breakdown are random events.

Figure 2 shows a histogram of the number of detected photons before decomposition for the 102 molecules. 

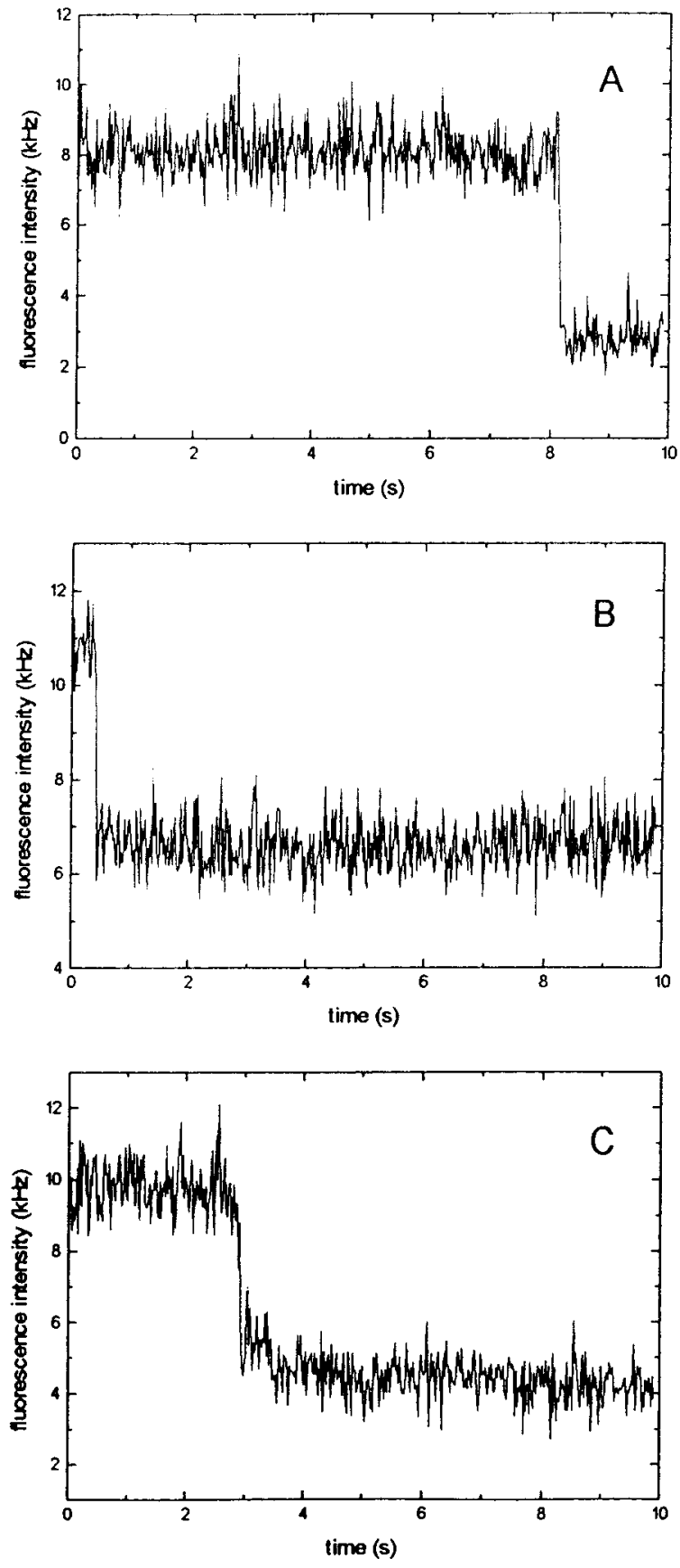

Figure 1. Three examples of decomposition of single molecules. The fluorescence intensity stays on a constant level as long as the molecule emits photons, but drops spontaneously to background level as the molecule dies.

Analysis of the number of photons from individual molecules suggests an exponential distribution; evaluating the histogram according to

$$
N(n)=N_{0} e^{-n / v}
$$

where $N$ is the number of molecules, $n$ is the number of detected photons, and $v$ is the death number yields $v=(9.8 \pm 1.0) \times$ $10^{3}\left(\chi^{2}=0.48\right)$ which is close to the mean number of photons for the 102 molecules of $1.0 \pm 0.1 \times 10^{4}$. Introducing two exponentially distributed populations results in $v_{1}=(10.8 \pm$ 4.2) $\times 10^{3}$ and $v_{2}=(2.6 \pm 2.8) \times 10^{3}\left(\chi^{2}=0.42\right)$ with the respective normalized amplitudes 0.65 and 0.35 .

In addition to the analysis of death numbers a histogram of the survival times of the molecules was made (Figure 3). An

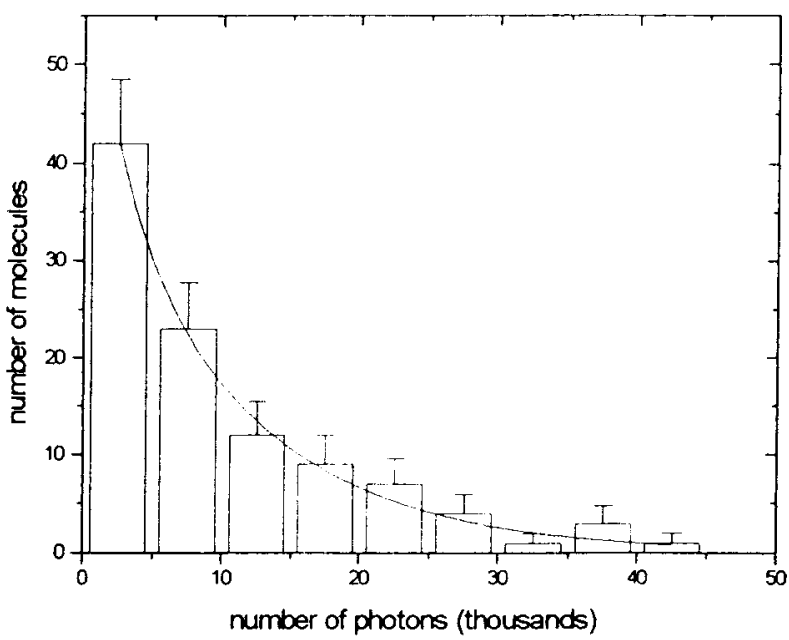

Figure 2. Histogram of the number of detected photons from the 102 molecules. A two-component exponential model gives two photon numbers of $v_{1}=(10.8 \pm 4.2) \times 10^{3}$ and $v_{2}=(2.6 \pm 2.8) \times 10^{3}$.

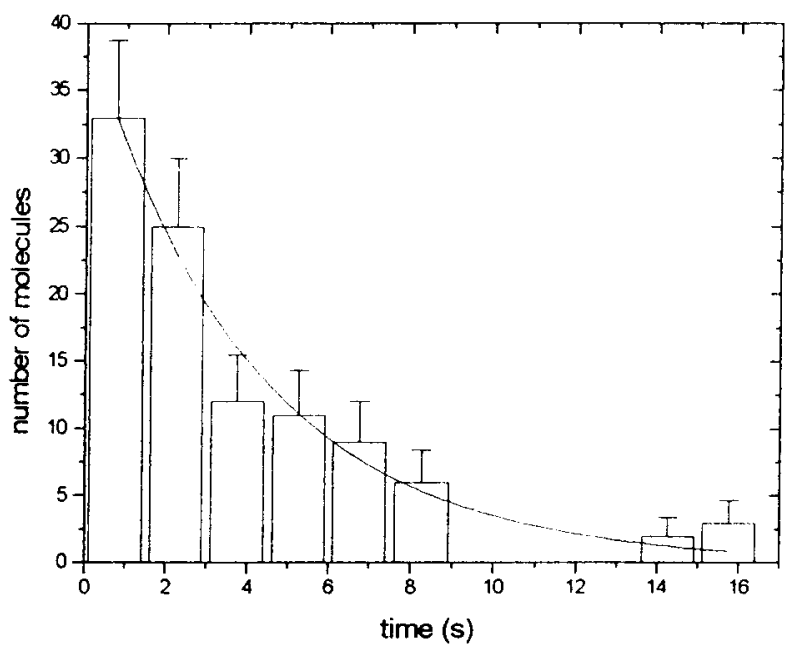

Figure 3. Histogram of the survival times of the 102 molecules with a single-exponential fit of $\tau_{\mathrm{s}}=4.1 \pm 0.5 \mathrm{~s}$ inserted.

evaluation according to

$$
N(t)=N_{0} e^{-t / \tau_{\mathrm{s}}}
$$

where $\tau_{\mathrm{s}}$ is the survival time, results in $\tau_{\mathrm{s}}=4.1 \pm 0.5 \mathrm{~s}\left(\chi^{2}=\right.$ 4.5 , solid line) whereas the average was $3.7 \pm 0.3 \mathrm{~s}$ (standard deviation of the mean).

Due to the limited statistics, the addition of a second exponential term to the model results in survival times with unreasonable variances.

Bulk Measurements. Different death numbers such as indicated in Figure 2 should be seen in the survival times in a bulk measurement. We incubated sample molecules on the streptavidin-coated coverslips in concentrations such that up to $100 / \mu \mathrm{m}^{2}$ were bound. Measurements of the fluorescence decay from many molecules simultaneously were carried out, of which one is shown (Figure 4). The decay rate is expected to be the same as for the distribution of the survival times of single molecules.

Because of the large number of molecules the disappearance of individual molecules cannot be seen. Only at low numbers was a step-type decay observed (not shown) indicating successive breakdown of individual molecules. With a laser intensity of $1 \times 10^{3} \mathrm{~W} / \mathrm{cm}^{2}$ the average fluorescence intensity from a single molecule is $3.4 \mathrm{kHz}$. The initial intensity $\left(I_{t=0}\right)$ in Figure 


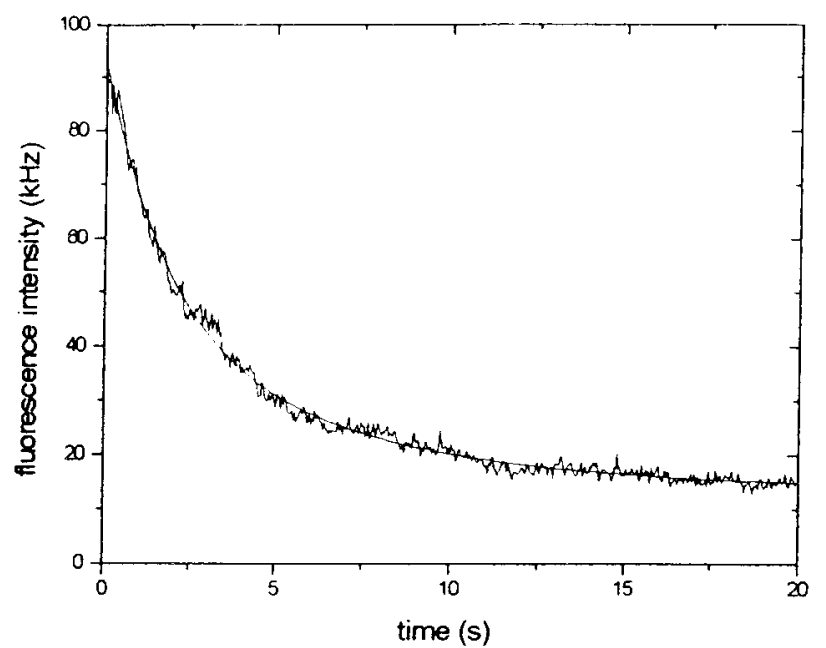

Figure 4. Fluorescence decay of 25-30 DNA molecules bound to the glass surface. Two survival times of $\tau_{1}=5.6 \pm 0.9 \mathrm{~s}$ and $\tau_{2}=1.6$ $\pm 0.3 \mathrm{~s}$ are evident.

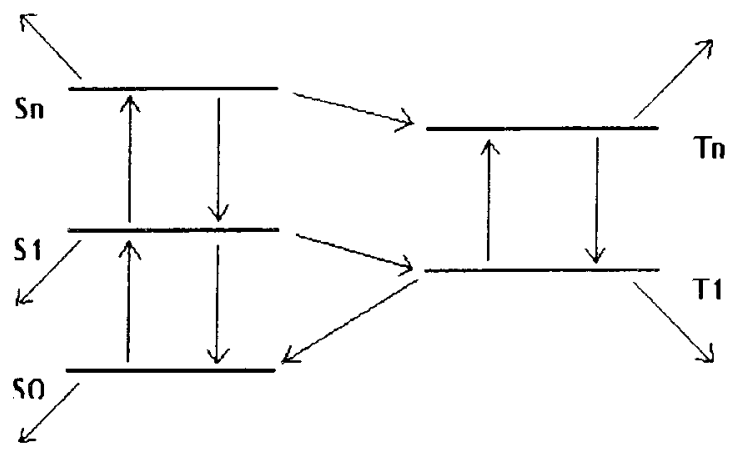

Figure 5. Kinetic scheme of the singlet-triplet interactions. Decomposition occurs from the $S_{1}$ or $T_{1}$ states, from the $S_{n}$ or $T_{n}$ states through ionization (at higher exitation intensities), or from any of the states through reaction with singlet oxygen.

4 is then due to $25-30$ molecules. A model with a single exponential results in $\tau_{\mathrm{s}}=3.1 \mathrm{~s}$ but does not fit the data sufficiently well $\left(\chi^{2}=3.2\right)$. Introducing an additional population gives two survival times of $\tau_{1}=5.6 \pm 0.9 \mathrm{~s}$ and $\tau_{2}=1.6 \pm$ $0.3 \mathrm{~s}$ with equal amplitudes $\left(\chi^{2}=2.1\right)$. The average survival times in such measurements were $\tau_{1}=8.1 \pm 3.1 \mathrm{~s}$ (standard deviation) and $\tau_{2}=1.7 \pm 0.6 \mathrm{~s}$ with the average normalized amplitudes 0.4 and 0.6 , respectively. The average number of molecules in the excitation volume was 22 , resulting in an average distance between neighboring molecules of $0.2 \mu \mathrm{m}$.

\section{Discussion}

When excited from the ground state the TMR molecule can reach several singlet as well as triplet states before returning to ground state by emission of a photon (Figure 5).

During photon emission, decomposition can occur from the $\mathrm{S}_{1}$ and the $\mathrm{T}_{1}$ state as a result of reactions with surrounding molecules due to increased reactivity of the excited fluorophore. Another mechanism is that the dye is excited to energetically higher singlet and triplet states $\mathrm{S}_{n}$ and $\mathrm{T}_{n}$ which in polar solvents such as water couple effectively to ionized states. ${ }^{12}$ This process becomes increasingly important at excitation intensities above $10^{4} \mathrm{~W} / \mathrm{cm}^{2}{ }^{12}$ a limit which is reached at the center of the Gaussian excitation profile.

Another possibility is the chemical bleaching of TMR through reaction with singlet oxygen. TMR can in this reaction be in any excited state or in the ground state. Singlet oxygen is formed from ground state (triplet) oxygen as ground-state oxygen quenches the majority of the dyes in the triplet state. ${ }^{13,14}$ Once the singlet oxygen is formed it may within its lifetime ( $2 \mu \mathrm{s}$ in water ${ }^{15}$ ) react with and destroy dye molecules in its vicinity. (With a diffusion of constant $D=10^{-9} \mathrm{~m}^{2} \mathrm{~s}^{-1}$, the average diffusion range is about $60 \mathrm{~nm}$, as compared to the average distance between molecules of $0.2 \mu \mathrm{m}$ (Figure 4)).

In a 3-dimensional bulk measurement the singlet oxygen can collide with and destroy any of the dyes in the investigated sample. In the single molecule study, however, once created the singlet oxygen diffuses away from the fluorophore and the probability of coming back to the starting point is negligible. The only way that singlet oxygen can destroy the fluorophore is that the destruction takes place directly after the singlet oxygen is formed and is still in the vicinity of the dye. Since the quantum yield of triplet formation for TMR is in the order of $10^{-312}$ and the average quantum yield of decomposition for the 102 molecules was $2 \times 10^{-6}$, a direct reaction has low probability for each singlet oxygen generated. The possibility that the reaction occurs during the 500 triplet entries typical for a molecule within its survival time can, however, not be excluded. In 3-dimensional bulk measurements, ${ }^{12}$ decomposition rates of the same magnitude are seen, despite the fact that each created singlet oxygen in this case has the possibility to collide with and destroy any dye in its vicinity. Thus, given that the dye is bleached by a singlet oxygen, it is likely that it is by a singlet oxygen created by the dye itself.

The complex molecule under study may undergo conformational fluctuations. ${ }^{2,3}$ In these fluctuations the molecule oscillates between two states, one in which the TMR sensor is close to a guanosin and one in which it is not. If the redox potentials are of the appropriate magnitudes an electron-transfer mechanism from the guanosin to the dye will compete with the photon emission from the excited singlet state in the case that TMR is close to guanosin. In this way the conformation in which TMR is close to guanosin will be less fluorescent. Measurement of the excited singlet state lifetime of TMR gives 4.06 and 1.36 ns for the normal and the quenched conformations, respectively. This results in a difference in the fluorescence intensity of the two states of a factor of 3. To confirm that an assumed decomposition step of a single molecule was not just a first observed step in a conformational fluctuation we continued to measure on the same spot for $30 \mathrm{~s}$ to see that the molecule was really dead. The $30 \mathrm{~s}$ control measurements also excluded the possibility of interpreting a high background intensity (Figure 1B) as the existence of an additional molecule.

Likely death numbers and survival times of TMR molecules are affected by the conformation of the DNA. Since none of the 102 molecules selected for analysis made a transition between the two states before breakdown, they were either in the low or the high fluorescent state the whole time. In the low fluorescent state TMR is likely to be intercalated between two bases and is therefore less available to surrounding molecules which in a collision with TMR could kill it. Thus the death number might be affected. Also the survival time will be affected by the particular conformation of the DNA. If TMR is in the low fluorescent state, it will in each cycle spend a shorter time (one-third) in the $\mathrm{S}_{1}$ state. Thus it will in each cycle have less probability to react with surrounding molecules or to enter the triplet state, which will result in a larger survival time.

Sorting the molecules in ascending fluorescence intensity shows that the death number is approximately constant for molecules with fluorescence intensity ranging from 3 to $8 \mathrm{kHz}$. Since the fluorescence intensity is related to the position of the 
molecule in the excitation volume, this shows no clear indication that centrally positioned molecules or high fluorescent conformations have lower death numbers. Still there exist several examples of low fluorescent molecules with survival times of $15 \mathrm{~s}$ and large death numbers. Therefore we cannot exclude the possibility that the lower death number originates from high fluorescent conformations that are not screened from oxygen, or from ionization of centrally positioned molecules.

A detailed analysis of the bulk measurements (Figure 4) has to take into account that the excitation intensity has a Gaussian distribution on the sample surface. The fluorescence intensity and thus the survival time of a molecule will be affected by its position on the excitation surface. The exact magnitude of the two survival times seen in such bulk measurements is therefore difficult to assess.

It is important to notice that-given a constant photon collection efficiency ${ }^{10}$ - unlike the survival time the death number is not dependent on the position of the molecule on the laser-exposed area. The death number is directly related to the number of completed excitation cycles of the dye and is not dependent on time.

To assess the significance of an indication of two components in exponential statistics, an index of separability is defined. ${ }^{16-18}$

$$
s=\frac{\left(v_{1}-v_{2}\right)}{\sqrt{\operatorname{var}\left(v_{1}\right)+\operatorname{var}\left(v_{2}\right)}}
$$

For the two death numbers the separability is equal to 1.64 (Figure 2). If the difference of the death numbers is subject to Gaussian fluctuations, a value of 1.64 represents a probability of 0.95 that a difference exists. ${ }^{16}$

We give here experimental support for the exponential distribution of death numbers according to

$$
I(n)=I_{0} e^{-n / v}
$$

where $n$ is the number of detected photons and $v$ is the death number. The exponential distribution cannot be obtained from bulk measurements and is the result of a constant probability of decomposition with each excitation. With a detection efficiency of about $2 \%$ the average number of emitted photons before decomposition for the 102 molecules in this study was $5 \times 10^{5}$ and the maximum number was $2 \times 10^{6}$. The twocomponent exponential decays indicate the existence of two populations with the different numbers of emitted photons $5 \times$ $10^{5}$ and $1 \times 10^{5}$ (Figure 2). A similar result, which was attributed to an inhomogeneous distribution of the environment, ${ }^{19}$ has been obtained on related systems where the critical photon count numbers before breakdown were measured on sulfurhodamine on PMMA surfaces. In our case the cromophore tetramethylrhodamine is not directly interacting with the surface (streptavidin) but is exposed to the solvent sitting on a $74 \mathrm{~nm}$ long DNA molecule anchored at the surface. The most likely explanations for the two death numbers seen in our data are the existence of two conformations which have different availability to oxygen, and the different probabilities of ionization due to the different positions of molecules in the excitation volume. Measuring single molecules with different excitation intensities and under different environmental conditions will further reveal the decomposition mechanisms discussed in this paper.

Acknowledgment. We thank Dr. Per Thyberg, Dr. Jerker Widengren, and Lars Edman for valuable discussions, Fredrik Tamsen for help with data collection, and Dr. Ilya Zelikman for help with streptavidin coating. This study was supported by grants from the Swedish Natural Science Research Council and Swedish Council for Engineering Sciences.

\section{References and Notes} 480

(1) Xie, X. S.; Trautman, J. K. Annu. Rev. Phys. Chem. 1998, 49, 441-

(2) Wennmalm, S.; Edman, L.; Rigler, R. Proc. Natl. Acad. Sci. U.S.A. 1997, 94, 10641-10646.

(3) Edman, L.; Mets, Ü.; Rigler, R. Proc. Natl. Acad. Sci. U.S.A. 1996, 93, 6710-6715.

(4) Eggeling, C.; Fries, J. R.; Brand, L.; Günther, R.; Seidel, C. A. M. Proc. Natl. Acad. Sci. U.S.A. 1998, 95, 1556-1561.

(5) Schmidt, Th.; Schütz, G. J.; Baumgartner, W.; Gruber, H. J.; Schindler, H. J. Phys. Chem. 1995, 99, 17662-17668.

(6) Wu, M.; Goodwin, P. M.; Ambrose, W. P.; Keller, R. A. J. Phys. Chem. 1995, 100, 17406-17409.

(7) Dörre, K.; Brakmann, S.; Brinkmeier, M.; Han, K.-T; Riebeseel, K.; Schwille, P.; Stephan, J.; Wetzel, T.; Lapczyna, M.; Stuke, M.; Bader, R.; Hinz, M.; Seliger, H.; Holm, J.; Eigen, M.; Rigler, R. Bioimaging 1997, $5,139-152$.

(8) Rigler, R.; Mets, Ü. Soc. Photo-Opt. Instrum. Eng. 1993, 1921, 239-248.

(9) Mets, Ü.; Rigler, R. J. Fluoresc. 1994, 4, 259-264.

(10) Rigler, R.; Mets, Ü.; Widengren, J.; Kask, P. Eur. Biophys. J. 1993 $22,169-175$

(11) Marquardt, D. J. Soc. Ind. Appl. Math. 1963, 11, 431-441.

(12) Eggeling, C.; Widengren, J.; Rigler, R.; Seidel, C. A. M. Anal. Chem. 1998, 70, 2651-2659.

(13) Widengren, J.; Mets, Ü.; Rigler, R. J. Phys. Chem. 1995, 99 , $13368-13379$.

(14) Widengren, J.; Rigler, R. Bioimaging 1996, 4, 149-157.

(15) Merkel, P. B.; Kearns, D. R. J. Am. Chem. Soc. 1972, 94, 72447253

(16) Sandor, T.; Conroy, M. F.; Hollenberg, N. K. Math. Biosci. 1970, $9,149-159$.

(17) Bajzer, Z.; Therneau, T. M.; Sharp, J. C.; Prendergast, F. G. Eur. Biophys. J. 1991, 20, 247-262.

(18) Köllner, M.; Wolfrum, J. Chem. Phys. Lett. 1992, 200, 199-204.

(19) Sanchez, E. J.; Novotny, L.; Holtom, G. R.; Xie, X. S. J. Phys. Chem. 1997, 101, 7019-7023. 